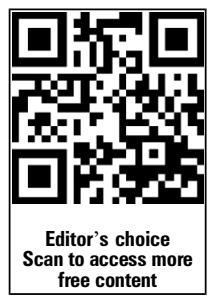

\section{Correspondence to}

Dr Arnab Seal, Leeds Community Healthcare NHS Trust and Leeds

Teaching Hospitals NHS Trust,

Children's Services,

Wortley Beck Health Centre,

Ring Road, Lower Wortley,

Leeds, West Yorkshire

LS12 5SG, UK.

arnab.seal@nhs.net

Received 10 April 2013

Revised 19 May 2013

Accepted 21 May 2013

Published Online First

21 June 2013

\title{
Fifteen-minute consultation on the infant with a large head
}

\section{Arnab Seal}

\section{ABSTRACT}

An infant with a large head (2.5 SDs above normal for weight and gender or above 99.6th centile for age) is a common clinical presentation. Usually, it is due to benign isolated macrocephaly or familial macrocephaly (FM) where some close family members are similarly affected $^{1}$; neither condition requires any further intervention. However, there are a few important underlying causes the clinician needs to actively consider and investigate when indicated before reassuring parents. These considerations include whether there is any associated developmental disorder or suggestion of a syndromic association or evidence of raised intracranial pressure (ICP).

\section{CLINICAL APPROACH}

In most instances, a careful history and examination either clarifies the diagnosis; for example, an ex-preterm child developing hydrocephalus. In other instances, it provides pointers for further investigation and management, for example, arranging genetic tests in a child presenting with macrocephaly and multiple cafe au lait spots suggesting the possible diagnosis of neurofibromatosis (NF) Type 1.

The following considerations are helpful.

1. Is the head actually large? The clinician should accurately measure and plot occipito-frontal circumference (OFC) using appropriate non-stretch tape measure. The OFC extends from the most prominent part of the glabella to the most prominent posterior area of the occiput or the largest measureable circumference. The OFC can be affected by thick hair and cranial bone deformations or hyperostosis. Height and weight should be plotted simultaneously as the OFC may be within normal centiles, but significantly discordant with body growth. If OFC is within normal centiles, but the head seems apparently large, then consider late intrauterine
Box 1 Conditions associated with higher risk of presenting with a large head in infancy

History of other immediate family member/s having asymptomatic large head

- Ex-preterm baby with history of intraventricular haemorrhage

- Previous history of subdural haemorrhage

- Neonatal meningitis

- Presence of non-cranial congenital anomalies can increase risk of brain anomalies and/or hydrocephalus, for example, spina bifida

- Family history of genetic syndromes associated with macrocephaly for example, neurofibromatosis type 1

- Relevant family history of developmental problems, for example, autism or learning disability

growth retardation, skeletal dysplasias for example, osteogenesis imperfecta, and conditions causing frontal bossing, for example, rickets.

2. Are there any serial measurements? Often the birth OFC and subsequent measurements are available from neonatal records and parent-held child health surveillance records. Is the OFC crossing centiles upwards? This does not necessarily

Table 1 Signs and symptoms of raised intracranial pressure (ICP) in infancy

\begin{tabular}{ll}
\hline Symptoms of raised ICP & Signs of raised ICP \\
\hline Irritability & Bulging fontanelle \\
High-pitched cry & Splayed sutures \\
Poor feeding & OFC crossing centiles \\
Vomiting & Sunsetting eyes \\
Decreased alertness & Prominent scalp veins \\
Developmental delay & Recent onset squint \\
Seizures & Lower limb spasticity \\
& Hyperreflexia \\
& Papilloedema
\end{tabular}

OFC, occipito-frontal circumference. 


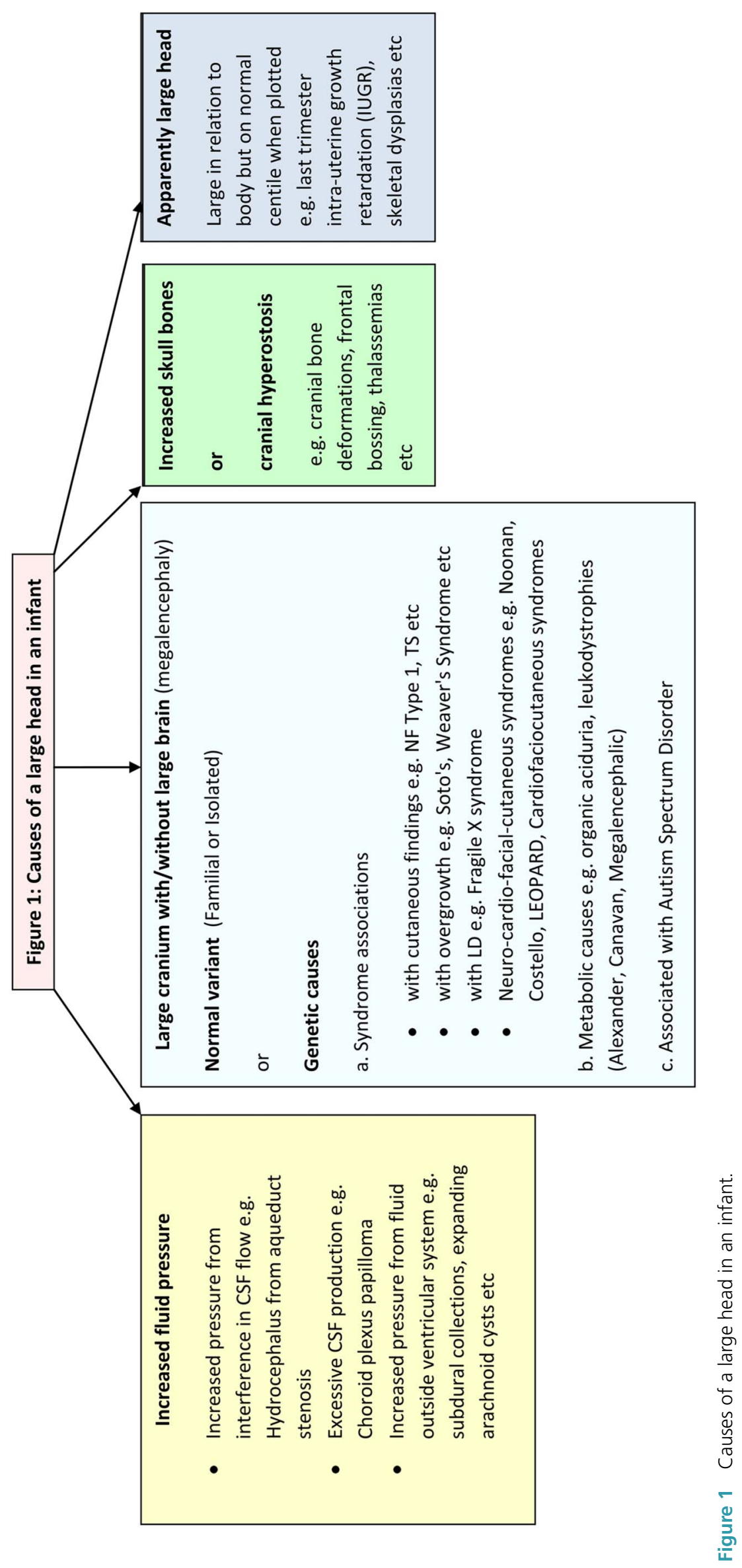




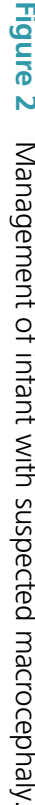
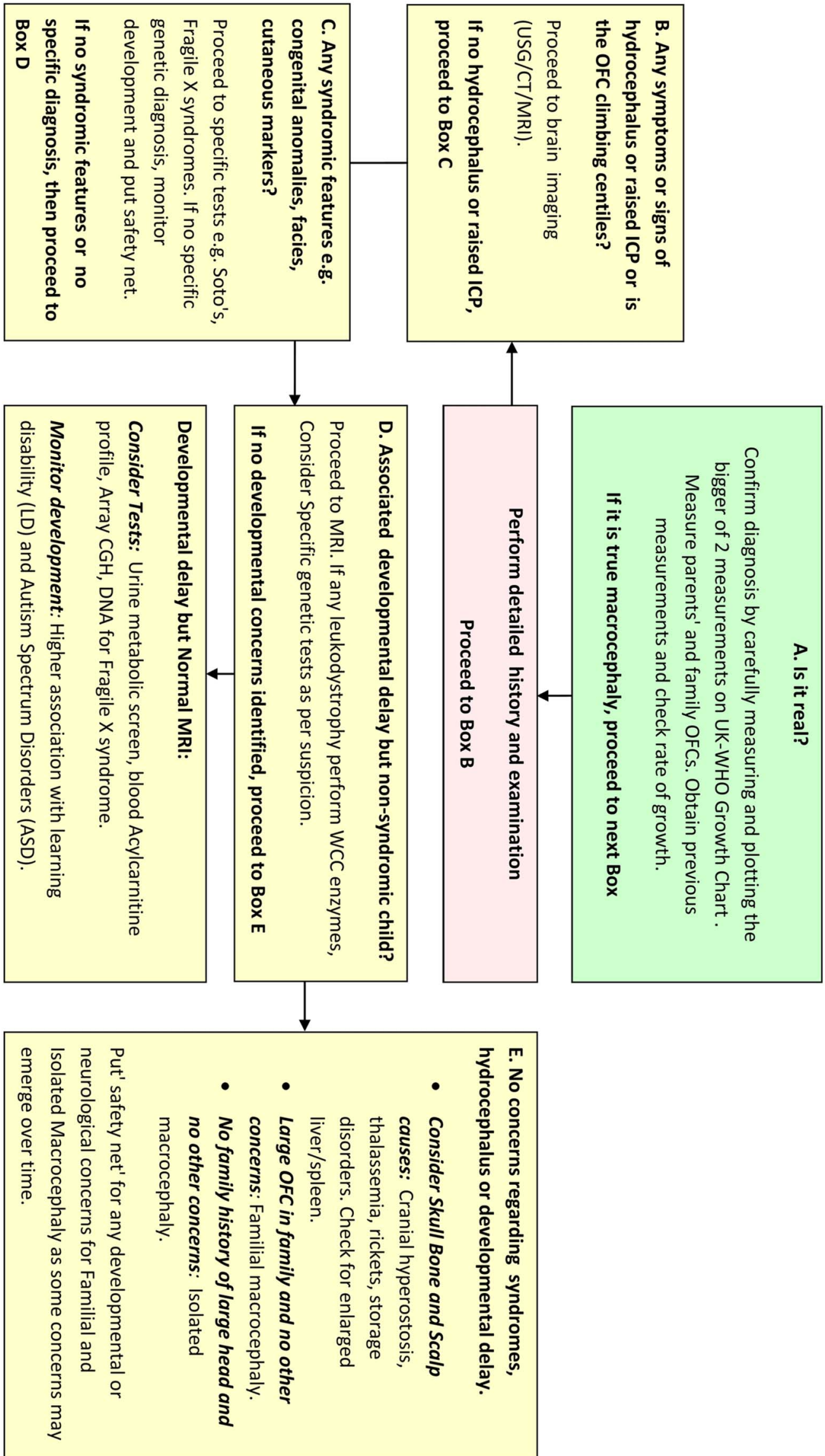
indicate a pathological cause as it may occur in benign $\mathrm{FM} /$ megalencephaly but should raise concern regarding raised ICP.

3. Are there any high-risk factors that are known to be associated with underlying causes of a large head in infancy? See box 1.

4. Are there any signs or symptoms of raised ICP? See table 1. If there is clinical suspicion of raised ICP, then proceed to neuroimaging, preferably MRI Brain. It is worth noting that all the signs and symptoms outlined are not always present.

5. Is there any history of developmental delay/disorder, seizures, loss of skills, behavioural change or neurological problem in the child? Take a specific developmental history and check developmental levels. Beware of the 'smiley child' who appears 'bright' but is not doing much. Remember, responsive social smile is achieved by 6-8 weeks age!

6. Measure parental and family (sibling, grandparents) head sizes where possible and plot on appropriate charts. The commonest cause for a large head is FM. If the child has no neurological symptoms or signs, has normal development, no signs of raised ICP, has normal systemic examination and the $\mathrm{OFC}$ is not climbing centiles, then reassure carers. If the OFC is crossing centiles, it still can be FM, but rule out raised ICP by neuroimaging. DeMyer's criteria for diagnosis of FM are absence of evidence of a syndrome, normal radiographic study of the brain and a parent or sibling with macrocephaly, or macrocephaly that can be traced through several generations. ${ }^{2}$ Discuss the diagnosis with the family and put a 'safety net' of symptoms and signs of raised ICP, seizures, learning delay or developmental concerns for carers to monitor.
7. A thorough examination specifically looking for any known causes of macrocephaly (see figure 1)

- dysmorphism

- unusual growth patterns, for example, increased growth in Soto's Syndrome

- neurocutaneous signs, for example, NF- 1, Tuberous sclerosis

- coarse facies or any signs of storage disorders like mucopolysaccharidoses

- full neurological examination including checks for focal deficits, co-ordination and fundus examination

- signs of raised ICP (see Box 1). Neuroimaging will usually identify the cause of raised ICP, for example, hydrocephalus, intracranial space occupying lesions (expanding cysts or tumours) or subdural collections

- hepatosplenomegaly suggesting storage disorders or haematological disorders, for example, thalassemias

Causes (see figure 1): The aetiology of macrocephaly can be divided into genetic and non-genetic causes. ${ }^{3}$

Management: Follow the algorithm in figure 2 from A to $\mathrm{E}$ to investigate and manage accordingly.

Competing interests None.

Provenance and peer review Commissioned; internally peer reviewed.

\section{REFERENCES}

1 Day RE, Scutt WH. Normal children with large heads-benign familial megalencephaly. Arch Dis Child 1979;54:512-17.

2 DeMyer W. Megalencephaly: types, clinical syndromes, and management. Pediatr Neurol 1986;2:321-8.

3 Williams CA, Dagli A, Battaglia A. Genetic disorders associated with macrocephaly. Am J Hum Genet 2008;146A:2023-37. 